\title{
Value of Free-Run Electromyographic Monitoring of Extraocular Cranial Nerves during Expanded Endonasal Surgery (EES) of the Skull Base
}

\author{
Parthasarathy D. Thirumala ${ }^{1,4}$ Santhosh Kumar Mohanraj ${ }^{1}$ Miguel Habeych ${ }^{1}$ Kelley Wichman ${ }^{1}$ \\ Yue-fang Chang ${ }^{1}$ Paul Gardner ${ }^{1}$ Carl Snyderman ${ }^{1,3}$ Donald J. Crammond ${ }^{1}$ Jeffrey Balzer ${ }^{1,2}$
}

${ }^{1}$ Department of Neurological Surgery, University of Pittsburgh, Pittsburgh, Pennsylvania, USA

2 Department of Neuroscience, University of Pittsburgh, Pittsburgh, Pennsylvania, USA

${ }^{3}$ Department of Otolaryngology, University of Pittsburgh, Pittsburgh, Pennsylvania, USA

${ }^{4}$ Department of Neurology, University of Pittsburgh, Pittsburgh,

Pennsylvania, USA

J Neurol Surg Rep 2013;74:43-50.

\begin{abstract}
Address for correspondence Parthasarathy D. Thirumala, MD, MS, Center for Clinical Neurophysiology, Department of Neurological Surgery, UPMC Presbyterian - Suite B-400, 200 Lathrop Street, Pittsburgh, PA 15213, USA (e-mail: thirumalapd@upmc.edu).
\end{abstract}

\begin{abstract}
Keywords

- electromyography

- lower cranial nerves

- expanded endonasal surgery

Objective To evaluate the value of free-run electromyography (f-EMG) monitoring of extraocular cranial nerves (EOCN) III, IV, and VI during expanded endonasal surgery (EES) of the skull base in reducing iatrogenic cranial nerve (CN) deficits.

Design We retrospectively identified 200 patients out of 990 who had at least one EOCN monitored during EES. We further separated patients into groups according to the specific CN monitored. In each CN group, we classified patients who had significant (SG) f-EMG activity as Group I and those who did not as Group II.

Results A total of 696 EOCNs were monitored. The number of muscles supplied by EOCNs that had SG f-EMG activity was 88 , including CN III $=46, C N I V=21$, and CN $\mathrm{VI}=21$. There were two deficits involving CN VI in patients who had SG f-EMG activity during surgery. There were 14 deficits observed, including $\mathrm{CNIII}=3, \mathrm{CN} I V=2$, and $\mathrm{CN}$ $\mathrm{VI}=9$ in patients who did not have SG f-EMG activity during surgery.

Conclusions f-EMG monitoring of EOCN during EES can be useful in identifying the location of the nerve. It seems to have limited value in predicting postoperative neurological deficits. Future studies to evaluate the EMG of EOCN during EES need to be done with both f-EMG and triggered EMG.
\end{abstract}

\section{Introduction}

Extraocular cranial nerve (EOCN) electromyographic monitoring during conventional skull base surgeries has been used to reduce the incidence of postoperative neurological deficits by detecting impending cranial nerve $(\mathrm{CN})$ injury. ${ }^{1-6}$ Free-run EMG (f-EMG) is a continuous recording of EMG activity characterized as spikes, bursts, and neurotonic discharges

received

December 13, 2012

accepted after revision

March 5, 2013

published online

June 13, 2013

during surgery secondary to unintended activation of the muscles supplied by the $\mathrm{CN}$. Neurotonic discharges ${ }^{7}$ during f-EMG recordings have been a reliable predictor of postoperative paralysis after skull base surgery. Triggered EMG (t-EMG) is a compound muscle action potential (CMAP) in response to voluntary stimulation of CNs during skull base surgery. The change in the threshold of stimulation to elicit a $\mathrm{CMAP}^{8}$ during facial nerve surgery may be a predictor of

(c) 2013 Georg Thieme Verlag KG Stuttgart · New York
DOI http://dx.doi.org/ 10.1055/s-0033-1346975. ISSN 2193-6358. 
postoperative $\mathrm{CN}$ paralysis. f-EMG and t-EMG responses can be recorded during surgery to localize EOCN. ${ }^{4}$ To date it has been difficult to correlate all or some type of f-EMG activity to predict postoperative deficits. Diplopia following intraoperative dissection of EOCN is a known complication. This leads to loss of stereoscopic vision and often requires monoocular occlusion, leading to secondary amblyopia., ${ }^{5,6}$ Hence, f-EMG and t-EMG can be very valuable if its utilization allows for prediction and prevention of postoperative EOCN paralysis. Various techniques have also been used for placement and recording both f-EMG and t-EMG discharges. These include placement of bipolar needles manually, or single-shafted bipolar needles using orbital ultrasound ${ }^{4}$ or image guidance. ${ }^{1}$ Surface recording using pad electrodes ${ }^{9}$ and ring electro$\operatorname{des}^{6,10}$ in the muscles have also been attempted.

Expanded endonasal surgery (EES) is a minimally invasive technique for skull base surgery that involves the use of an endoscope and complex neuronavigational systems with neurosurgery and otolaryngology working together during all phases of surgery. ${ }^{11-15}$ Using the principles of EES, we are able to access the ventral skull base from the crista galli to odontoid in the sagittal plane and as lateral as the middle fossa with minimal residual postoperative complications. ${ }^{16}$ Based on the location of the tumor, the approach can be classified into transcribriform, transdorsum sellae, and transclival. Although minimally invasive, EES still carries a potential risk for injury to neurovascular structures, including the internal carotid arteries, anterior cerebral arteries, and CNs III-XII $^{12-14}$ depending on the approach and location of the tumor. Our primary aim of this paper was to evaluate the value of f-EMG monitoring of EOCN during EES in reducing iatrogenic EOCN deficits.

\section{Materials and Methods}

\section{Study Design}

We retrospectively reviewed all consecutive cases of EES at our institution between 2000 and 2008. The total number of patients monitored during EES was 990. Patients who did not have intraoperative EMG monitoring data or patients who did not have EMG monitoring due to technical reasons were excluded from this study. Patients who did not have a documentation of postoperative neurologic status were also excluded. Using these criteria, a total of 200 patients were identified as having at least one EOCN monitored during the operation. Included patients in our study for each EOCN were CN III: 153 , CN IV: 135 , and CN VI: 187 . One patient might have more than one nerve monitored. This study was approved by the quality assurance committee for retrospective review of data on human subjects at the University of Pittsburgh.

\section{Neurophysiologic Monitoring}

Continuous f-EMG activity was recorded using pairs of subdermal needle electrodes placed $1 \mathrm{~cm}$ apart in the orbit in the location of the muscle groups innervated by EOCN. Our technique involves a simple approach to placing bipolar needles after gently moving the globe away from the orbit using our fingers: place two needles with the sharp edges directed toward the bony aspect of the orbit, and repeat the procedure for all the other nerves. f-EMG monitoring of CN III involved placement in the medial rectus muscle, $\mathrm{CN}$ IV by placement in the superior oblique muscle, and $\mathrm{CN}$ VI by placement in the lateral rectus. The needle length was $13 \mathrm{~mm}$ long with a diameter of $0.4 \mathrm{~mm}$ manufactured by Rhythmlink (Columbia, South Carolina, USA). Physician oversight and interpretation was performed using a combined onsite and remote model utilized by University of Pittsburgh Medical Center (UPMC). In all cases, a board-certified neurophysiologist was onsite and immediately available for interpretation and consultation, and physician (neurologist) oversight, supervision, and interpretation were performed in-person or remotely. The oversight physician provided supervision to four to five cases simultaneously on average, with a maximum of eight cases.

\section{Anesthesia}

Short-acting neuromuscular junction blocking medications were used for intubation. No additional paralytic agent was administered, particularly during f-EMG monitoring. Neuromuscular junction blockade was tested by using a standard train of four twitches. The patient was maintained using a balanced anesthetic technique using inhalational and IV agents during the procedure.

\section{Alarm Criteria}

No detectable f-EMG activity was considered baseline in each case. Detection of nerve manipulation, compression, stretch, and/or permanent injury was based on changes from baseline recordings. All instances of $\mathrm{f}$-EMG activity, regardless of type (spikes, bursts, neurotonic discharges), were made audible and were also immediately reported to the surgeon and recorded in the patient record. Henceforth, this EMG activity will be called "EMG alerts." SG f-EMG activity when present for prolonged periods of time, or prolonged activities with small interruptions for a single lower $\mathrm{CN}$, were reported as one alert. Recordings were made continuously during all stages of surgery after incision. The settings were bandpass filters set at 3 to $1,000 \mathrm{~Hz}$, with a gain of 5,000, a time base of 100 milliseconds, and a sensitivity of 50 microvolts. All EMG activity was made audible to the surgeon via a loudspeaker connected to the collection unit. In our study we did not consider EMG activity during irrigation as significant.

\section{Medical Record Review}

Medical records of all patients who had EES were reviewed to determine if any new neurologic deficits were present after the surgical procedures. The medical records were reviewed independently without knowledge of their intraoperative EMG changes. Any new postoperative deficits were considered to be iatrogenic intraoperative injuries. Deficits were classified as transient or permanent. Transient deficits had documented evidence in medical records of complete improvement to baseline. Permanent deficits were defined as those that did not improve to baseline in subsequent followup visits. All EOCN deficits were documented by 
ophthalmologists. Similarly, the intraoperative records were reviewed to look for EMG discharges; all 200 were reviewed independently without the knowledge of postoperative neurologic outcome.

\section{Demographic and Patient Data}

We compiled all demographic, diagnostic, and surgical information on the patients who had any EOCN EMG monitoring. These data were analyzed with each individual CN. Each EOCN was segregated; patients who had significant f-EMG activity during the surgery were classified as Group I and patients who did not have significant f-EMG activity during the surgery were classified as Group II. The need for CN monitoring was determined by the surgeon and the neurophysiologist based on the pathology and a perceived risk of injury to EOCN. The purpose of the comparative analysis was to identify variables, which were significantly different between the two groups. Data were presented as mean \pm standard deviation for continuous variables and proportion for categorical variables. Chi-square tests or Fisher exact tests were used for categorical variable comparisons. The analyses were performed by SAS 9.13.

\section{Data Analysis}

EMG alerts were annotated in the technologist's log for all instances where EOCN f-EMG activity was observed. EMG alerts were considered significant if EMG activity information was conveyed to the surgeons. This was followed by estimating the number of EMG alerts per nerve, which is provided to the surgeon during the case. Correlation to any EMG alerts and postoperative neurological deficits was performed. For data analysis, the number of nerves monitored for each $\mathrm{CN}$ was calculated by adding unilateral as "one" and bilateral as "two" nerves monitored. Furthermore, the data from the two groups were tabulated to compare and correlate the number of transient and permanent deficits in the patients who were monitored.

\section{Results}

\section{Demographic and Patient Data}

The total number of patients who had at least one EOCN monitored was 200 . Of 200 patients, $44 \%$ were male and $56 \%$ female. All the patients had surgery between 2000 and 2008. Twenty-five percent of patients underwent surgery before 2006 and 75\% underwent surgery after 2006. There is no significant difference between the demographic or surgical information between the two groups. Meningioma was the most common diagnosis in both groups. The other diagnoses included pituitary tumor, chordoma, angiofibroma, chondrosarcoma, and others. Transclival was the most common EES approach, followed by transellar and transplanum. More than one approach was performed in some cases. During such instances, we referred to the primary approach. Further subgroup analysis was performed to identify the differences in demographic and patient data for each specific EOCN. No clinically significant variables like age, sex, procedure, diagnosis, number of stages, or presence of neurological deficits was present when both groups were compared (-Table $\mathbf{1}$ ).

\section{Monitoring EOCN}

Monitoring of at least one EOCN was performed in 200 patients. CN III was monitored in 153 patients. CN IV was monitored in 135 patients. CN VI was monitored in 187 patients (-Table $\mathbf{2}$ ).

\section{SG f-EMG Activity and EOCN Deficits}

A total of 696 EOCNs were monitored in 200 patients. The percentage incidence of EOCN deficit in nerves with significant activity is $2.27 \%$ and percentage incidence of EOCN deficit in nerves without significant activity is $2.30 \%$. The details of individual EOCNs monitored, SG f-EMG activities, and their postoperative nerve deficits are as follows (-Table 2): 88 cranial nerves had significant f-EMG activity in the muscles supplied by them.

1. CN III: A total of 153 patients had 219 nerves monitored during surgery. There were 46 nerves (21\%) that displayed SG f-EMG activity. No deficits were present in the group with SG activity, and three permanent EOCN deficits were observed in the group without SG f-EMG activity.

2. CN IV: A total of 135 patients had 192 nerves monitored during surgery. There were 21 nerves (11\%) that displayed SG f-EMG activity. No postoperative EOCN deficits were observed in the group with SG f-EMG activity and two postoperative EOCN deficits in the group without SG f-EMG activity.

3. CN VI: A total of 187 patients had 285 nerves monitored during surgery. There were 18 nerves (7\%) that displayed

Table 1 Demographic and Patient Data of Monitored Patients

\begin{tabular}{|l|l|l|}
\hline Number of patients monitored & 200 & $100 \%$ \\
\hline Sex & 88 & $44 \%$ \\
\hline Male & 112 & $56 \%$ \\
\hline Female & \multicolumn{2}{|l|}{} \\
\hline Surgical approach & 51 & $26 \%$ \\
\hline Transellar & 91 & $46 \%$ \\
\hline Transclival & 13 & $6.50 \%$ \\
\hline Transplanum & 12 & $6.00 \%$ \\
\hline Transcavernous & 12 & $6.00 \%$ \\
\hline Transpterygoidal & 21 & $10.50 \%$ \\
\hline Others & \multicolumn{2}{|l}{} \\
\hline Year of surgery & 50 & $25 \%$ \\
\hline 2000-2005 & 150 & $75 \%$ \\
\hline 2006-2008 & 43 & $22 \%$ \\
\hline Diagnosis & 25 & $13 \%$ \\
\hline Meningioma & 37 & $18.50 \%$ \\
\hline Chordoma & 6 & $3.00 \%$ \\
\hline Pituitary tumor & 77 & $6.00 \%$ \\
\hline Chondrosarcoma & $37.50 \%$ \\
\hline Angiofibroma & \multicolumn{2}{|l}{} \\
\hline Others & 12 \\
\hline
\end{tabular}


Table 2 Data Indicating the Cranial Nerve Monitored and Postoperative Outcome

\begin{tabular}{|l|l|l|l|l|l|l|l|l|l|l|}
\hline Cranial nerves & III & IV & VI & V & VII & IX & X & XI & XII \\
\hline Total number of patients monitored & 153 & 135 & 187 & & 16 & 39 & 32 & 35 & 14 & 32 \\
\hline Total number of nerves monitored & 219 & 192 & 285 & & 24 & 63 & 55 & 61 & 24 & 59 \\
\hline Side monitored & & & & & & & & & & \\
\hline Right & 43 & 40 & 41 & & 3 & 9 & 5 & 5 & 2 & 2 \\
\hline Left & 44 & 38 & 48 & 5 & 6 & 4 & 4 & 2 & 3 \\
\hline Both & 66 & 57 & 98 & & 8 & 24 & 23 & 26 & 10 & 27 \\
\hline Total number of patients with significant activity & 39 & 20 & 18 & & 1 & 10 & 6 & 6 & 2 & 2 \\
\hline Number of cranial nerves with significant activity & 46 & 21 & 21 & & 1 & 15 & 8 & 10 & 4 & 3 \\
\hline Total cranial nerve deficits in patients with significant activity & 0 & 0 & 2 & & 0 & 0 & 0 & 0 & 0 & 0 \\
\hline Transient & 0 & 0 & 0 & 0 & 0 & 0 & 0 & 0 & 0 \\
\hline Permanent & 0 & 0 & 2 & 0 & 0 & 0 & 0 & 0 & 0 \\
\hline Total number of patients without significant activity & 114 & 115 & 169 & & 15 & 29 & 26 & 29 & 12 & 30 \\
\hline Number of cranial nerves without significant activity & 173 & 171 & 264 & 23 & 48 & 47 & 53 & 20 & 56 \\
\hline Total cranial nerve deficits in patients without significant activity & 3 & 2 & 9 & 0 & 0 & 1 & 1 & 0 & 1 \\
\hline Transient & 0 & 1 & 2 & 0 & 0 & 0 & 0 & 0 & 0 \\
\hline Permanent & 3 & 1 & 7 & 0 & 0 & 1 & 1 & 0 & 1 \\
\hline
\end{tabular}

SG f-EMG activity. There were two permanent EOCN deficits in patients who had SG f-EMG activity. A total of two transient and seven permanent CN VI deficits were observed in the group without SG f-EMG activity.

\section{Clinical Information on EOCN Deficits}

A total of 608 nerves did not display SG f-EMG discharges. Among these nerves, a total of $14 \mathrm{CN}$ deficits were noted. Three deficits were seen in CN III, two in CN IV, and nine in CN VI (-Table 7). Patient's initials are marked in parentheses.

- Patient (SR) underwent surgery for removal of pituitary adenoma. This patient did have permanent right CN III, IV, and VI deficit after her first stage of EES. No SG f-EMG activity was observed from any $\mathrm{CN}$ monitored.

- Patient (SD) underwent surgery for removal of chondrosarcoma. The patient had permanent right CN III deficits and temporary right CN IV deficit after her first stage of EES. No SG f-EMG activity was present in either of the nerves or the CN VI monitored.

- Patient (PR) had a left CN III deficit with no SG f-EMG activity in CN III or IV.

- There were nine CN VI deficits, in which two CN VI deficits were transient and seven were permanent. One patient had CN III, IV deficits as described above. Another patient had bilateral CN VI palsy, with one temporary and one permanent deficit. The other six deficits were all only $\mathrm{CN}$ VI. The common diagnosis in this group with CN VI deficits is pituitary tumor; the common approach to the tumor has been transellar approach. There were five patients with $\mathrm{CN}$ VI deficits after stage 1 , three CN VI deficits after stage 2, and one after stage 4 .
The incidence, mean and standard deviation, and range of significant activity during surgery in each individual CN group is also calculated and shown in the table (-Table 3 ).

Further analysis was performed by comparing the group with significant activity and the group without significant activity during EOCN monitoring. There were no significant differences in their age, sex, period of surgery, number of stages, procedures, neurological deficits, laterality, or EMG alerts for CN III, IV, and VI (-Tables 4-6).

In addition to monitoring EOCN, we also monitored lower CNs (VII, IX, X, XI, XII) in some cases during the same EES procedures (-Table 2 ). No CN deficits were seen in patients who had SG f-EMG activity. Three lower cranial deficits were seen in one patient who also had CN VI palsy. No SG f-EMG activity was present in any one of the nerves.

\section{Discussion}

EOCN EMG monitoring has been used during traditional skull surgery to identify the EOCN and prevent postoperative EOCN deficits. f-EMG is a continuous recording of EMG activity characterized by spikes, bursts, and neurotonic discharges secondary to unintended activation of the $\mathrm{CN}$ supplying extraocular musculature. Neurotonic discharges, ${ }^{7}$ a highfrequency discharge f-EMG, have been predictive of postoperative paralysis after skull base surgery. The f-EMG activity can reflect the proximity of CNs during EES, hence providing value in the operating field and potentially reducing the incidence of iatrogenic injury.

The incidence of EOCN deficit between patients who had SG f-EMG activity during surgery (2.27\%) and patients who did not have SG f-EMG activity during surgery (2.30\%) is not 
Table 3 Data Show Incidence of Free-run Electromyographic Activity in the Extraocular Cranial Nerve

\begin{tabular}{|c|c|c|c|}
\hline Cranial nerve & III & IV & VI \\
\hline Number of patients monitored & 153 & 135 & 187 \\
\hline Number of nerves monitored & 219 & 192 & 285 \\
\hline Number of nerves with significant activity & 46 & 21 & 21 \\
\hline Incidence of significant activity & $21 \%$ & $11 \%$ & $7 \%$ \\
\hline Mean and standard deviation for EMG alerts per nerve & $3.4 \pm 3.08$ & $2.10 \pm 1.49$ & $2.22 \pm 1.59$ \\
\hline Median and range for EMG alerts per nerve & $3(1-14)$ & $1(1-6)$ & $2(1-6)$ \\
\hline Free-run EMG activity observed in patients with CN deficits & 0 & 0 & 2 \\
\hline
\end{tabular}

Abbreviations: CN, cranial nerve; EMG, electromyelography.

significantly different. Manipulation of the EOCN by dissecting instruments and drilling can evoke f-EMG activity including bursts, spikes, or neurotonic discharges. There appears to be no correlation between the type of manipulation and the discharges that were recorded. ${ }^{7}$ It also appears that the f-EMG activity provides continuous feedback on the location of the nerve. This feedback resulted in a more cautious approach to dissection of the tumor and hence may prevent neurological deficits in EOCNs that had SG f-EMG activity during surgery. It is recognized that sudden complete transection of a nerve does not produce any f-EMG activity. ${ }^{17,18}$ It is also possible that the absence of the f-EMG alerts provides a false sense of security, promoting a more aggressive resection resulting in increased risk of nerve injury.

We regarded any change in f-EMG activity above baseline as significant activity and immediately relayed the EMG alert to the surgery team. The f-EMG alerts were very useful in indicating the proximity to a $\mathrm{CN}$. The mean number of EMG alerts of each EOCN varied from 2.1 to 3.4 during each procedure (-Table $\mathbf{3}$ ). It is unclear if the amount of EMG alerts conveyed to the surgeon is of any value in predicting $\mathrm{CN}$ deficit. This might be secondary to the fact that, once significant activity is relayed to the surgeon, he or she will take a conservative approach to safeguard the nerve. The incidence of SG f-EMG activity for each CN, a percentage of total nerves with significant activity to the number of nerves monitored, were $21 \%, 11 \%$, and $7 \%$ for CN III, IV, and VI, respectively. There was no correlation between the incidence of SG f-EMG activity and postoperative EOCN deficits.

In our series, $21 \%$ of monitored CN III had SG f-EMG activity during the procedure. CN III generated most alerts when compared with CNs IV and VI. CN VI was also the most common nerve monitored. Incidentally, this was the nerve with the smallest number of EMG alerts (7\%) during surgery,

Table 4 Statistical Analysis of Different Variables in Cranial Nerve III

\begin{tabular}{|c|c|c|c|c|c|c|}
\hline \multicolumn{7}{|l|}{ Cranial nerve III } \\
\hline & & \multicolumn{2}{|c|}{$\mathrm{SG}=\mathrm{no}$} & \multicolumn{2}{|c|}{$\mathrm{SG}=$ yes } & \multirow[b]{2}{*}{$p$ Value } \\
\hline & & $n$ & row $\%$ & $n$ & row $\%$ & \\
\hline \multirow[t]{2}{*}{ Sex } & Female & 51 & 71.83 & 20 & 28.17 & \\
\hline & Male & 59 & 74.68 & 20 & 25.32 & 0.693 \\
\hline \multirow[t]{2}{*}{ Stage } & 1 & 85 & 72.03 & 33 & 27.97 & \\
\hline & 2 to 8 & 24 & 77.42 & 7 & 22.58 & 0.547 \\
\hline \multirow[t]{3}{*}{ Procedure } & TRANSCLIVAL & 38 & 65.52 & 20 & 34.48 & \\
\hline & TRANSSELLAR & 36 & 75.00 & 12 & 25.00 & \\
\hline & Others & 36 & 81.82 & 8 & 18.18 & 0.174 \\
\hline \multirow[t]{2}{*}{ Year of surgery } & $2000-2005$ & 17 & 68.00 & 8 & 32.00 & \\
\hline & $2006-2008$ & 93 & 74.40 & 32 & 25.60 & 0.509 \\
\hline \multirow[t]{5}{*}{ ND } & No & 170 & 78.70 & 46 & 21.30 & \\
\hline & Yes & 3 & 100 & 0 & 0.00 & 1.000 \\
\hline & & \multicolumn{2}{|c|}{$\mathrm{SG}=\mathrm{no}$} & \multicolumn{2}{|c|}{$\mathrm{SG}=$ yes } & \\
\hline & & mean & $S D$ & mean & $S D$ & $p$ Value \\
\hline & Age & 51.21 & 17.88 & 44.91 & 16.79 & 0.055 \\
\hline
\end{tabular}

Abbreviations: ND, neurological deficit; SD, standard deviation; SG, significant activity. 
Table 5 Statistical Analysis of Different Variables in Cranial Nerve IV

\begin{tabular}{|c|c|c|c|c|c|c|}
\hline \multicolumn{7}{|l|}{ Cranial nerve IV } \\
\hline & & \multicolumn{2}{|c|}{$\mathrm{SG}=\mathrm{no}$} & \multicolumn{2}{|c|}{$\mathrm{SG}=$ yes } & \multirow[b]{2}{*}{$p$ Value } \\
\hline & & $N$ & row $\%$ & $n$ & row \% & \\
\hline \multirow[t]{2}{*}{ Sex } & female & 54 & 87.10 & 8 & 12.90 & \\
\hline & male & 52 & 81.25 & 12 & 18.75 & 0.369 \\
\hline \multirow[t]{2}{*}{ Stage } & 1 & 83 & 84.69 & 15 & 15.31 & \\
\hline & 2 to 8 & 22 & 81.48 & 5 & 18.52 & 0.768 \\
\hline \multirow[t]{3}{*}{ Procedure } & TRANSCLIVAL & 37 & 77.08 & 11 & 22.92 & \\
\hline & TRANSSELLAR & 39 & 90.70 & 4 & 9.30 & \\
\hline & others & 30 & 85.71 & 5 & 14.29 & 0.198 \\
\hline \multirow[t]{2}{*}{ Year of surgery } & $2000-2005$ & 14 & 82.35 & 3 & 17.65 & \\
\hline & $2006-2008$ & 92 & 84.40 & 17 & 15.60 & 0.733 \\
\hline \multirow[t]{5}{*}{ ND } & no & 169 & 88.95 & 21 & 11.05 & \\
\hline & yes & 2 & 100.00 & 0 & 0.00 & 1.000 \\
\hline & & \multicolumn{2}{|c|}{ SG $=$ no } & \multicolumn{2}{|c|}{$\mathrm{SG}=$ yes } & \\
\hline & & mean & $S D$ & mean & $S D$ & $p$ Value \\
\hline & Age & 48.34 & 17.28 & 49.25 & 21.56 & 0.835 \\
\hline
\end{tabular}

Abbreviations: ND, neurological deficit; SD, standard deviation; SG, significant activity.

as well. Anatomical location and the intracranial course of CN III, IV, and VI can explain this phenomenon. But the exact reason for this difference could be related to the size and direction of tumor growth, involvement of the CNs, and approach to the tumor removal. This is supported by the absence of cross-correlation of the incidence of SG f- EMG discharges between EOCNs. It is interesting to note that $\mathrm{CN}$ VI had the most deficits and the lowest level of SG f-EMG activity. EES involves a midline approach to tumors through the nose. It is possible that minimal manipulation of the tumor resulted in EMG activity during the procedure. The increased alerts could be a protective effect against excessive

Table 6 Statistical Analysis of Different Variables in Cranial Nerve VI

\begin{tabular}{|c|c|c|c|c|c|c|}
\hline \multicolumn{7}{|l|}{ Cranial Nerve VI } \\
\hline & & \multicolumn{2}{|c|}{$\mathrm{SG}=\mathrm{no}$} & \multicolumn{2}{|c|}{ SG $=$ yes } & \multirow[b]{2}{*}{$p$ Value } \\
\hline & & $n$ & row $\%$ & $n$ & row $\%$ & \\
\hline \multirow[t]{2}{*}{ Sex } & female & 78 & 91.76 & 7 & 8.24 & \\
\hline & male & 89 & 89.90 & 10 & 10.10 & 0.663 \\
\hline \multirow[t]{2}{*}{ Stage } & 1 & 126 & 91.30 & 12 & 8.70 & \\
\hline & 2 to 8 & 40 & 88.89 & 5 & 11.11 & 0.570 \\
\hline \multirow[t]{3}{*}{ Procedure } & TRANSCLIVAL & 76 & 88.37 & 10 & 11.63 & \\
\hline & TRANSSELLAR & 46 & 95.83 & 2 & 4.17 & \\
\hline & others & 45 & 90.00 & 5 & 10.00 & 0.411 \\
\hline \multirow[t]{2}{*}{ Year of surgery } & $2000-2005$ & 29 & 82.86 & 6 & 17.14 & \\
\hline & $2006-2008$ & 138 & 92.62 & 11 & 7.38 & 0.100 \\
\hline \multirow[t]{5}{*}{ ND } & no & 255 & 93.07 & 19 & 6.93 & \\
\hline & yes & 9 & 81.82 & 2 & 18.18 & 0.190 \\
\hline & & \multicolumn{2}{|c|}{$\mathrm{SG}=\mathrm{no}$} & \multicolumn{2}{|c|}{$\mathrm{SG}=$ yes } & \\
\hline & & mean & $S D$ & mean & $S D$ & $p$ Value \\
\hline & Age & 47.75 & 18.82 & 43.09 & 17.70 & 0.329 \\
\hline
\end{tabular}

Abbreviations: ND, neurological deficit; SD, standard deviation; SG, significant activity. 
Table 7 Clinical Extraocular Cranial Nerve Deficits in Individual Patients

\begin{tabular}{|c|c|c|c|c|c|c|c|}
\hline Patient \# & Age & Sex & Diagnosis & Surgery & $\mathrm{CN}$ monitored & Side monitored & Nerve deficit \\
\hline 1 & 62 & Male & Clival chordoma & Transclival & VI & Bilateral & VI left \\
\hline 2 & 69 & Female & Meningioma & Transclival & $\mathrm{VI}$ & Right & VI right \\
\hline 3 & 34 & Female & Meningioma & Transclival & $\mathrm{VI}$ & Bilateral & VI right \\
\hline 4 & 48 & Female & Pituitary adenoma & Transellar & $\mathrm{VI}$ & Bilateral & VI bilateral \\
\hline 5 & 64 & Female & Pituitary adenoma & Transcavernous & IV\&VI & Right side in both & $\begin{array}{l}\text { IV \& VI right } \\
\text { in both }\end{array}$ \\
\hline 6 & 22 & Male & Pituitary adenoma & $\begin{array}{l}\text { Transclival } \\
\text { Transellar-zone } 4\end{array}$ & $\mathrm{VI}$ & Right & VI right \\
\hline 7 & 13 & Male & Angiofibroma & Transpterygoidal & $\mathrm{VI}$ & Bilateral & VI right \\
\hline 8 & 13 & Male & Angiofibroma & Transpterygoidal & IV & Bilateral & IV left \\
\hline 9 & 66 & Male & Pituitary adenoma & Transellar & III & Left & III left \\
\hline 10 & 58 & Female & Pituitary adenoma & Transellar & III & Right & III right \\
\hline 11 & 53 & Male & Pituitary adenoma & $\begin{array}{l}\text { Transclival, } \\
\text { Transplanum- } \\
\text { Transclival }\end{array}$ & $\mathrm{VI}$ & Bilateral & VI left \\
\hline 12 & 30 & Female & Pituitary adenoma & Transellar & $\mathrm{VI}$ & Right & VI right \\
\hline 13 & 59 & Female & Pituitary adenoma & Transcavernous & $\mathrm{III \& VI}$ & Bilateral in both & $\begin{array}{l}\text { III \&VI right } \\
\text { in both }\end{array}$ \\
\hline
\end{tabular}

Abbreviation: CN, cranial nerve.

manipulation or just an epiphenomenon due to the anatomical nature of their CN III course. We believe that the surgical techniques are modified secondary to the EMG alerts.

f-EMG has been used to identify EOCN and prevent postoperative deficits. Schlake et al used both f-EMG and t-EMG to identify EOCN III and IV and to predict postoperative deficits. ${ }^{4}$ A total of $18 \mathrm{CNs}$ were monitored; however, not all nerves could be identified using t-EMG. There was no correlation between EMG activity and neurological deficits. A similar study by Sekhar et al demonstrated the usefulness of t-EMG in identifying EOCN. ${ }^{5}$ There was no relationship between EMG activity and postoperative nerve deficits. Sekiya et al used tEMG to monitor 22 EOCN; no f-EMG was performed. ${ }^{10}$ Changes in the threshold of stimulation to obtain a CMAP response may be useful to predict postoperative nerve injury. ${ }^{8}$ Loss of CMAP during surgery can indicate transection of a nerve during surgery. Kawaguchi et al found a correlation between increased threshold and postoperative nerve injury. ${ }^{2}$ It appears t-EMG can be used to identify EOCN during traditional skull base surgery. The f-EMG, irrespective of classification, does not correlate with postoperative EOCN deficits. Similarly, in our study we found no direct correlation between EMG activity and postoperative EOCN deficits.

We had a total of 14 EOCN deficits in patients who did not have SG f-EMG activity. There were nine CN VI deficits. Two $\mathrm{CN}$ VI deficits were transient and seven were permanent. The common diagnosis in this group with CN VI deficits is pituitary tumor, the common approach to the tumor has been transellar approach. There were five patients with CN VI deficits after stage 1 , three CN VI deficits after stage 2 , and one after stage 4 . Previous surgery and radiotherapy would have made dissection of the region of the tumor very difficult due to adhesions. The deficits also may be explained by inadvertent sharp transection of the monitored nerve during surgery. ${ }^{18,19}$ Radiation could have also modified the EMG response secondary to inadvertent stimulation. Spontaneous EMG activity will occur if the nerve is subjected to stretching, traction, or injury. If the nerve is transected abruptly, there will be only brief or no EMG activity as described by Harper et al. ${ }^{20}$ Lack of f-EMG activity should not provide a false sense of security about the state or proximity of the $\mathrm{CN}$. Using t-EMG to localize the CNs during tumor resection may potentially help prevent nerve injuries in these types of cases. These results also emphasize the limitation of just using fEMG during EES. We have modified our approach for monitoring and are consistently using t-EMG in addition to f-EMG in our practice to identify EOCN and to prevent iatrogenic $\mathrm{CN}$ deficits.

We further analyzed the data to see if any particular variable is significantly different between two groups. Variables considered were gender, stage of surgery, year of surgery, and surgical approach. We considered age because older patients are more prone to succumb to postoperative morbidity and there is a high risk for stroke, which can lead to various nerve deficits. The year the surgery took place is considered because we found a learning curve effect on EES with SSEP monitoring. ${ }^{21}$ None of the variables was significantly different in either group (-Tables 4-6).

This study did have limitations. Only f-EMG was monitored during the surgery and no t-EMG monitoring was performed. f-EMG is clearly an unintentional evoked EMG response and hence it is less specific. Additionally, we only monitored cases 
that were at high risk for $\mathrm{CN}$ injury. In our entire series, we had 990 patients who underwent EES. We did have CN deficits in patients who were not monitored, so the current strategy to identify patients for f-EMG might have to expand to include more patients. Our analysis included deficits in patients recorded after surgery. It is possible some deficits were not clearly documented. The technique of placing needles in the extraocular muscles to record EMG is not standardized. It is possible that our technique of placing needles is not very sensitive to record f-EMG and hence had limited value in predicting postoperative deficits.

\section{Conclusions}

- EOCN f-EMG monitoring during EES can provide useful information to identify the location of the $\mathrm{CN}$. This may also be used to alert the surgeon to make adjustments during the procedure, which can prevent EOCN injury.

- EOCN f-EMG seems to have limited value in predicting postoperative neurological deficits. Absence of EMG activity during the procedure should not provide a false sense of security about the state or proximity of the CN.

- If and when possible, CMAP with t-EMG should be elicited to check the integrity of EOCN. Further studies to evaluate the EMG of EOCN during EES procedures need to be done with both f-EMG and t-EMG.

- We advocate a comprehensive approach to neurophysiological monitoring during EES, including somatosensoryevoked potentials, f-EMG, t-EMG of CNs II-XII, brain stem auditory-evoked potentials, and electroencephalogram depending on the location of the neural structures at risk and the approach being used.

\section{Disclosures}

No disclosures are pertinent to the paper.

\section{Note}

No grants are pertinent to this paper.

\section{References}

1 Alberti O, Sure U, Riegel T, Bertalanffy H. Image-guided placement of eye muscle electrodes for intraoperative cranial nerve monitoring. Neurosurgery 2001;49(3):660-663, discussion 663-664

2 Kawaguchi M, Ohnishi H, Sakamoto T, et al. Intraoperative electrophysiologic monitoring of cranial motor nerves in skull base surgery. Surg Neurol 1995;43(2):177-181

3 Schlake HP, Goldbrunner R, Milewski C, et al. Technical developments in intra-operative monitoring for the preservation of cranial motor nerves and hearing in skull base surgery. Neurol Res 1999;21(1):11-24
4 Schlake HP, Goldbrunner R, Siebert M, Behr R, Roosen K. IntraOperative electromyographic monitoring of extra-ocular motor nerves (Nn. III, VI) in skull base surgery. Acta Neurochir (Wien) 2001;143(3):251-261

5 Sekhar LN, Møller AR. Operative management of tumors involving the cavernous sinus. J Neurosurg 1986;64(6):879-889

6 Sekiya T, Hatayama T, Iwabuchi T, Maeda S. Intraoperative recordings of evoked extraocular muscle activities to monitor ocular motor nerve function. Neurosurgery 1993;32(2): 227-235, discussion 235

7 Romstöck J, Strauss C, Fahlbusch R. Continuous electromyography monitoring of motor cranial nerves during cerebellopontine angle surgery. J Neurosurg 2000;93(4):586-593

8 Isaacson B, Kileny PR, El-Kashlan HK. Prediction of long-term facial nerve outcomes with intraoperative nerve monitoring. Otol Neurotol 2005;26(2):270-273

9 Fukaya C, Katayama Y, Kasai M, Kurihara J, Yamamoto T. Intraoperative electro-oculographic Monitoring for Skull Base Surgery. Skull Base Surg 2000;10(1):11-15

10 Sekiya T, Hatayama T, Iwabuchi T, Maeda S. A ring electrode to record extraocular muscle activities during skull base surgery. Acta Neurochir (Wien) 1992;117(1-2):66-69

11 Kassam A, Carrau RL, Snyderman CH, Gardner P, Mintz A. Evolution of reconstructive techniques following endoscopic expanded endonasal approaches. Neurosurg Focus 2005;19(1):E8

12 Kassam A, Snyderman CH, Mintz A, Gardner P, Carrau RL. Expanded endonasal approach: the rostrocaudal axis. Part I. Crista galli to the sella turcica. Neurosurg Focus 2005;19(1):E3

13 Kassam A, Snyderman CH, Mintz A, Gardner P, Carrau RL. Expanded endonasal approach: the rostrocaudal axis. Part II. Posterior clinoids to the foramen magnum. Neurosurg Focus 2005;19(1):E4

14 Kassam AB, Gardner P, Snyderman C, Mintz A, Carrau R. Expanded endonasal approach: fully endoscopic, completely transnasal approach to the middle third of the clivus, petrous bone, middle cranial fossa, and infratemporal fossa. Neurosurg Focus 2005; 19(1):E6

15 Kassam AB, Snyderman C, Gardner P, Carrau R, Spiro R. The expanded endonasal approach: a fully endoscopic transnasal approach and resection of the odontoid process: technical case report. Neurosurgery 2005;57(1, Suppl):E213, discussion E213

16 Gardner PA, Kassam AB, Snyderman CH, et al. Outcomes following endoscopic, expanded endonasal resection of suprasellar craniopharyngiomas: a case series. J Neurosurg 2008;109(1):6-16

17 Harner SG, Daube JR, Ebersold MJ. Electrophysiologic monitoring of facial nerve during temporal bone surgery. Laryngoscope 1986; 96(1):65-69

18 Harner SG, Daube JR, Ebersold MJ, Beatty CW. Improved preservation of facial nerve function with use of electrical monitoring during removal of acoustic neuromas. Mayo Clin Proc 1987; 62(2):92-102

19 Nelson KR, Vasconez HC. Nerve transection without neurotonic discharges during intraoperative electromyographic monitoring. Muscle Nerve 1995;18(2):236-238

20 Harper CM. Intraoperative cranial nerve monitoring. Muscle Nerve 2004;29(3):339-351

21 Thirumala PD, Kassasm AB, Habeych M, et al. Somatosensory evoked potential monitoring during endoscopic endonasal approach to skull base surgery: analysis of observed changes. Neurosurgery 2011;69(Suppl 1):64-76 